\title{
Health Canada proposes nutrition label changes
}

$\mathrm{H}$ ealth Canada has proposed changes to nutrition labels on food to help Canadians make healthy food choices, but some critics say the reforms don't go far enough.

According to a Health Canada press release, the proposed changes would make it easier for Canadians to read labels, compare similar foods, understand how much sugar items contain and make more informed food choices. Health Minister Rona Ambrose stated that Canada is "breaking new ground" with its proposals for identifying amounts of sugars in food, which include percent daily values and grouping sugars in the list of ingredients.

But Bill Jeffery, national coordinator for the Center for Science in the Public Interest, says the proposed labels would be hard to distinguish from the current labels. "Over the past year, the government deep-sixed almost every constructive proposal for fixing food labels, including easy-to-understand front-of-package nutrition labelling and a separate line for added or free sugars," Jeffery wrote in an email.

On Twitter, obesity expert Dr. Yoni Freedhoff, medical director of the Bariatric Medical Institute in Ottawa, also noted the lack of a separate line for added sugars. In a blog post, he wrote that the proposed label reforms do not go far enough and that giving the food industry five years to implement the changes (after a public consultation and 18 months to publish final changes) could only be explained as "pandering to industry."

Health Canada, however, referred to the proposed changes, which are based on feedback from more than 10000 Canadians, as a "major step forward" in its press release. Proposed changes to the nutrition facts table include adding the amount of potassium, percent daily value for sugars and a footnote suggesting that a value of $5 \%$ or less is "a little" and $15 \%$ or more "is a lot." Minor

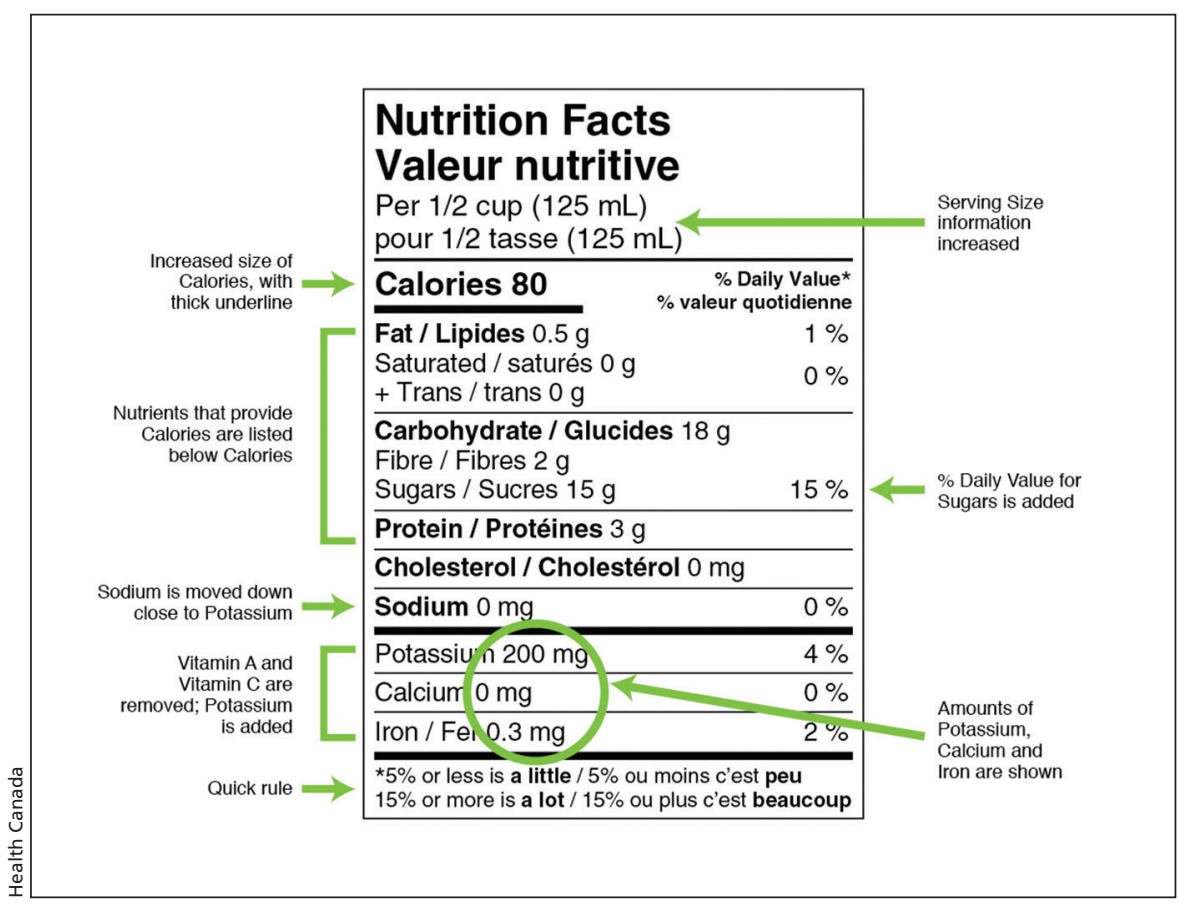

Proposed changes to the nutrition facts table include the addition of the amount of potassium and percent daily value of sugars.

changes to enhance readability have been proposed for the list of ingredients, including using a dark font on a white or neutral background, grouping sugarbased ingredients and separating items with bullets.

Health Canada is proposing that listed serving sizes should be consistent on similar foods and be realistic, reflecting more closely the portions people actually eat. For foods that can be measured, serving sizes would be shown as common household measurements, such as cups or teaspoons, paired with its metric equivalent in grams or millilitres. Serving sizes for foods eaten in pieces would be shown as either the number of pieces (e.g., per 8 crackers) or fraction of total (e.g., per $1 / 4$ frozen pizza), along with its metric equivalent. Certain foods will have serving sizes that reflect how much people typically eat, such as sliced bread (2 slices).

In a statement on behalf of the Cen- ter for Science in the Public Interest, Jeffery wrote that the proposed label reforms would have a "puny impact" on public health, the biggest shortcoming being the failure to propose "userfriendly front-of-package labelling" based on a nutrition score amplified by traffic light colour-coding.

"Better nutrition labelling could be very effective at improving public health, lengthening lifespans, improving Canadians' quality of life, saving scarce health care dollars, and compressing the period of illness and disability at the end of life," wrote Jeffery. "That matters mightily, and should matter a lot more than food companies' reluctance to calculate new nutrition numbers and reprint labels, which additional costs are basically the same no matter what the labeling regulations require." — Roger Collier, CMAJ

CMAJ 2015. DOI:10.1503/cmaj.109-5099 